\title{
Diversity of honey bee Apis mellifera subspecies (Hymenoptera: Apoidae) from the Western Highlands of Cameroon based on morpho-biometry
}

\author{
Fotso KPR. ${ }^{1,2}$ 2* Costa-Maia FM. ${ }^{3}$ Keambou TC. ${ }^{4}$ Baenyi SP..$^{5}$ Defang FH. ${ }^{1}$ Manjeli Y. ${ }^{1}$ \\ Teguia A. ${ }^{1}$ Tchoumboué J. ${ }^{1}$
}

\author{
${ }^{I}$ Faculty of Agronomy and Agricultural Sciences, University of Dschang, Cameroon \\ ${ }^{2}$ Government Technical and Professional High School of Agriculture in Yabassi, Cameroon \\ ${ }^{3}$ Department of Animal Science, Federal University of Technology Paraná, Dois Vizinhos, Brazil \\ ${ }^{4}$ Bioscience Eastern and Central Africa - International Livestock Research Institute, Nairobi, Kenya \\ ${ }^{5}$ Faculty of Agricultural Sciences and Environment, Evangelical University in Africa, Democratic \\ Republic of the Congo
}

*Corresponding author: fotsokenmognepr@gmail.com

Article history; Received: January $5^{\text {th }}$ 2021; Revised: May 10 ${ }^{\text {th }}$ 2021; Accepted: May $28^{\text {th }} 2021$

\begin{abstract}
The study was carried out from August to September 2016 in the Western Highlands of Cameroon. The main objective was to contribute to a better understanding of biodiversity of honeybees for their preservation and genetic improvement. To attain this, samples of 420 workers bees belonging to Apis mellifera subspecies were obtained from 14 localities in the study zone. Body measurements in $\mathrm{mm}$ gave the following values: honey bee length $(10.98 \pm 0.06)$, abdomen length $(6.71 \pm 0.03)$, width of the yellow band on the second $\left(2^{\text {nd }}\right)$ abdominal tergite $(1.48 \pm 0.01)$, width of fourth $\left(4^{\text {th }}\right)$ abdominal tergite $(1.32 \pm 0.01)$, length of cover hair on the fifth $\left(5^{\text {th }}\right)$ abdominal tergite $(0.29 \pm 0.00)$, antenna length $(4.27 \pm 0.02)$, proboscis length $(4.39 \pm 0.11)$, length of nervure $\mathrm{A}$ $(0.60 \pm 0.00)$, length of nervure B $(0.25 \pm 0.00)$, discoidal shift $(-0.13 \pm 0.01)$, anterior right wing length $(9.31 \pm$ $0.03)$, anterior right wing width $(3.14 \pm 0.01)$, posterior right wing length $(6.38 \pm 0.03)$ and posterior right wing width $(1.69 \pm 0.01)$. A correlation coefficient significantly $(p<0.01)$ higher $(0.72)$ was obtained between the length of posterior right wing and the length of the anterior right wing. The cubital index is $2.36 \pm 0.04$. Populations of honeybees studied consist of three genetic types; genetic types 2 and 3 are closer together. The observed biodiversity suggests that honeybees constitute a natural resource with genetic variability needed for preservation and genetic improvement.
\end{abstract}

Keywords: Adansonii, Beekeeping, Biometric, Biometric index, Morphology

\section{Introduction}

Beekeeping is a lucrative activity and constitutes a source of employment and additional sustainable income for the beekeepers and the craftsmen making beehives (Fotso et al. 2014). Further, honeybees through their pollinating activity are crucial agents for crop productivity and food security worldwide. In Cameroon, no records indicate the presence of domesticated honeybees populations, and beekeepers used mostly local methods (fire) to harvest honey produced by wild colonies, which is a threat to honeybees because beehives and colonies are destroyed (Tchoumboué et al. 2001). A multitude of subspecies and ecotypes of honeybees are reported today (Bouga et al. 2011). However, honeybees of the Western Highlands of Cameroon are thought to belong to the same subspecies Apis mellifera adansonii (Tchoumboué et al. 2001; Fotso et al. 2014). This apparent observed homogeneity in tropical honeybees is rather due to a lack of diversity studies on honeybee populations in Central Africa (Paraïso et al. 2011). However, studies have shown the existence of two subspecies with geographical distribution extending from East to West Africa, with colonies of intermediate nature, sharing traits from many subspecies, namely A. mellifera jemenitica from Mali to Sudan and A. mellifera adansonii in the region covering Senegal, Benin, Nigeria, Cameroon and the Congo Bassin (Ruttner 1987). Similarly, the genetic study of different subspecies of honeybees in sub Saharan Africa has shown the presence of A. mellifera adansonii and A. mellifera nigritarium (Francoy et al. 2008). 
The observations made by Tchoumboué et al. (2011) and Fotso et al. (2014) indicate that, honeybee present in the Western Highlands of Cameroon is A. mellifera adansonii. They display three different features of honeybees: the yellow type, smaller and produce more honey; the black type, larger and produce less honey; the gray type, resulting from the crossing of the two previous ones. Studies on the morphometric characters of A. mellifera subspecies are therefore necessary for the characterization of honeybees, the preservation of their biodiversity and the sustainability of beekeeping production systems. The output of this study will (i) improve the state of knowledge about the honeybee of the Western Highlands of Cameroon, (ii) contribute to preserving biodiversity and develop appropriate genetic improvement programs. This study will be analysing the different honeybees' body measurements, their genetic variability, the structure of the population studied and the phylogenetic relationships of honeybees in the Western Highlands of Cameroon.

\section{Material and Methods}

\section{Study area and Choice of the localities}

The study was conducted in the Western Highlands of Cameroon. The average altitude varies from 700 to $1700 \mathrm{~m}$ with geographical coordinates $4^{\circ} 79^{\prime}-6^{\circ} 43^{\prime} \mathrm{NL}$ and $9^{\circ} 68^{\prime}-11^{\circ} 52^{\prime} \mathrm{EL}$.

The climate is of the Cameroonian type modified by the altitude. The temperature varies from 16 to 27 ${ }^{\circ} \mathrm{C}$, and the relative humidity between 49 and $97 \%$. The average rainfall is about $2000 \mathrm{~mm}$ ranging from 1700 to over $2500 \mathrm{~mm} / \mathrm{year}$ and divided into two seasons. The rainy season runs from midMarch to mid-November and the dry season from mid-November to mid-March. The orographic influences explain the microclimates observed in the region. The average annual insolation is 2500 hours.

The highlands forests are surrounded by grassy meadows with Sporobolus africanus. The main tree species present are : Celtis africana, Nuxia prunis, Rapanea sp, Syzigium standtii, Terstroemia polypeptala. In upland areas with accumulation of organic matter, the tree layer is represented by Albizia gummifera, Allophyllus bullatus, Pygeum africanum and Syzigium guineensis. The lower part of the highland meadows is often cultivated and the area is dominated by Hyparrhenia rufa, Sporobolus africanus and Melinis minutiflora. The plant species found at the foot of maneguba are: Lophira lanceolata, Cussonia barteri, Hymenocardia acida, Nauclea latifolia, Annonia chrysophylla and Terminalia glaucescens. The vegetation of the alluvial-colluvial valleys includes many plant species that are fond of imperfectly drained environments. These include: Andropogon gayanus, Digitaria abyssinica, Cyperus giganteus, Mitragyna stipulosa and Raffia palm. Some plant species are anthropogenic, such as resinous eucalyptus and kola trees, often associated with avocado and banana trees. In addition, there are some bushy legumes like, Calliandra sp are present and used as fodder.

With the support of the "House of Western Beekeepers" (HWB) and on the basis of the study conducted by Fotso et al. (2014), beekeepers with accessible apiaries and hives with moving frames placed about one meter from the ground were identified. Based on these criteria, a total of 14 localities were selected from the eight administrative division of the Western Region of Cameroon, caution been made to have the localities distributed across different altitudes.

\section{Choice of apiaries, hives and Worker honeybees' Collection}

In each locality, an apiary of at least 5 hives was chosen, according to its accessibility. In each apiary, 2 to 4 hives were selected and workers were collected. In each hive, 25 to 30 honey bee workers were collected (Toullec 2008). The workers were collected in jars and stored in ethanol at $95^{\circ} \mathrm{C}$. Measurements were made only on female workers, as males are haploid and are not representative in a honeybee's population (Toullec 2008).

\section{Morphometric Data Collection}

Information regarding morphometric characters were recorded on a suitable record as described by Francoy et al. (2008), Tofilski (2008), Amakpe (2010), Carreck et al. (2011), Oyerinde et al. (2012) and FAO (2013). The information included total length, abdomen length, width of the yellow band on the $2^{\text {nd }}$ abdominal tergite, width of the $4^{\text {th }}$ abdominal tergite, length of the hairiness of the hairy zone on the $5^{\text {th }}$ abdominal tergite, antenna length, proboscis length, length of nervure $\mathrm{A}$, length of nervure 
$\mathrm{B}$, length of right forewing, width of right forewing, discoidal shift, right hindwing length and right hindwing width. The body measurements were made using a Zeiss binocular microscope (Stemi 2000C), equipped with a camera connected to a computer thanks to Zen 2012 software (Zen 2012 SP2) with X40 magnification (Bouga et al. 2011; Meixner et al. 2013; Hadis et al. 2016).

The morphometric data allowed to establish the biometric indices as defined by Fresnaye (1965), Bouga et al. (2011), Carreck et al. (2011), Meixner et al. (2013) and Hadis et al. (2016). The cubital index (CI) was evaluated as the ratio of the length of the nervures A/B (Cornuet et al. 1975; Fresnaye 1981; Toullec 2008). The transgression displacement or discoidal index, according to the position of the discoidal point, the nature of the transgression was determined (Cornuet et al. 1975; Fresnaye 1981; Toullec 2008).

\section{Statistical Analysis}

The following statistical model was used:

$$
y_{i j}=\mu+\alpha_{i}+e_{i j}
$$

$\boldsymbol{\mu}$ : the Average of the population;

$\alpha_{i}$ : the Effect of the locality i;

$\mathbf{e}_{\mathbf{i j}}$ : the Residual error of each Bees $\mathrm{j}$ in the Locality $\mathrm{i}$.

The analysis of variance (ANOVA) allowed to evaluate the influence of the locality on the various body measurements. When there was significant difference the mean was separated using the Duncan multiple range test. The orientation and degree of association between the traits were determined using Pearson's correlation coefficients.

To assess genetic variability, principal component analysis (PCA) was performed on the basis of the 14 biometric characteristics.

To identify the different subspecies, a typology of honeybees was performed using discriminant factor analysis (DFA) based on the fourteen body measurements (FAO 2013). The construction of the phylogenetic tree following the hierarchical ascending classification (HAC) protocol has established phylogenetic relationships among populations (Carpentier 2007; Roux 2006).

The statistical analyses were performed using SPSS 21.0 and XLSTAT 2014 software.

\section{Results}

Body measurements of honeybees from the Western Highlands of Cameroon

Table 1 shows the sample size, mean values and coefficients of variation of honeybees' morphometric characteristics according to locations in the Western Highlands of Cameroon. 
Fotso et al, 2021. Gen. Biodiv. J: 5 (2): 1-11

Table 1: Number, mean values and coefficients of variation of honey bee morpho-metric characteristics according to locations in the Western Highlands of Cameroon

\begin{tabular}{|c|c|c|c|c|c|c|c|c|c|c|c|}
\hline \multirow[b]{2}{*}{ Localities } & \multirow[b]{2}{*}{$\mathrm{N}$} & \multicolumn{2}{|l|}{$\mathrm{Lb}(\mathrm{mm})$} & \multicolumn{2}{|l|}{ Lab (mm) } & \multicolumn{2}{|l|}{ Wyb (mm) } & \multicolumn{2}{|l|}{ Wab (mm) } & \multicolumn{2}{|l|}{$\operatorname{Lh}(\mathrm{mm})$} \\
\hline & & $\overline{\boldsymbol{X}}_{ \pm \text {S.E }}$ & $\mathrm{CV}(\%)$ & $\overline{\boldsymbol{X}}_{ \pm \text {S.E }}$ & $\mathrm{CV}(\%)$ & $\overline{\boldsymbol{X}}_{ \pm \text {S.E }}$ & CV $(\%)$ & $\overline{\boldsymbol{X}}_{ \pm \text {S.E }}$ & $\mathrm{CV}(\%)$ & $\overline{\boldsymbol{X}}_{ \pm \text {S.E }}$ & CV (\%) \\
\hline Santchou & 30 & $9.29 \pm 0.17^{\mathrm{f}}$ & 10.07 & $6.04 \pm 0.11^{\mathrm{f}}$ & 10.30 & $1.47 \pm 0.03^{\mathrm{bc}}$ & 11.29 & $1.16 \pm 0.05^{\mathrm{d}}$ & 27.71 & $0.26 \pm 0.01^{\text {ef }}$ & 24.37 \\
\hline Melia & 30 & $10.55 \pm 0.27^{\mathrm{de}}$ & 14.50 & $6.67 \pm 0.15^{\text {de }}$ & 12.61 & $1.44 \pm 0.06^{c}$ & 24.67 & $1.27 \pm 0.03^{\mathrm{bcd}}$ & 17.07 & $0.29 \pm 0.01^{\text {bddef }}$ & 23.13 \\
\hline Dschang & 30 & $10.50 \pm 0.23^{\text {cde }}$ & 9.51 & $7.20 \pm 0.09^{\mathrm{b}}$ & 9.25 & $1.55 \pm 0.02^{\mathrm{abc}}$ & 9.03 & $1.40 \pm 0.05^{\mathrm{b}}$ & 19.62 & $0.31 \pm 0.01^{\mathrm{ab}}$ & 27.57 \\
\hline Bamepa & 30 & $10.50 \pm 0.23^{\mathrm{de}}$ & 12.18 & $6.34 \pm 0.09^{f}$ & 8.33 & $1.53 \pm 0.08^{\mathrm{abc}}$ & 29.63 & $1.26 \pm 0.04^{\mathrm{cd}}$ & 21.54 & $0.34 \pm 0.01^{\mathrm{a}}$ & 18.65 \\
\hline Bamessingue & 30 & $11.62 \pm 0.23^{\mathrm{ab}}$ & 11.15 & $7.75 \pm 0.10^{\mathrm{a}}$ & 7.52 & $1.59 \pm 0.02^{\mathrm{ab}}$ & 8.99 & $1.63 \pm 0.05^{\mathrm{a}}$ & 17.58 & $0.30 \pm 0.00^{\mathrm{abcd}}$ & 17.57 \\
\hline Santa & 30 & $10.44 \pm 0.23^{\mathrm{de}}$ & 12.07 & $6.89 \pm 0.09^{\mathrm{bcd}}$ & 7.40 & $1.49 \pm 0.02^{\mathrm{abc}}$ & 10.58 & $1.38 \pm 0.03^{\mathrm{bc}}$ & 13.88 & $0.29 \pm 0.00^{\text {bcde }}$ & 15.48 \\
\hline Famla'a & 30 & $11.87 \pm 0.27^{\mathrm{a}}$ & 12.76 & $7.02 \pm 0.13^{\mathrm{bc}}$ & 10.78 & $1.62 \pm 0.04^{\mathrm{a}}$ & 13.70 & $1.37 \pm 0.05^{\mathrm{bc}}$ & 23.40 & $0.31 \pm 0.01^{\mathrm{abc}}$ & 22.67 \\
\hline Koutaba & 30 & $10.23 \pm 0.22^{\mathrm{e}}$ & 12.20 & $6.24 \pm 0.09^{f}$ & 8.05 & $1.47 \pm 0.02^{\mathrm{bc}}$ & 9.95 & $1.20 \pm 0.04^{\mathrm{d}}$ & 20.20 & $0.27 \pm 0.01^{\text {cdef }}$ & 23.16 \\
\hline Banka & 30 & $11.98 \pm 0.16^{\mathrm{a}}$ & 7.36 & $6.36 \pm 0.09^{\mathrm{cf}}$ & 8.59 & $1.48 \pm 0.03^{\mathrm{bc}}$ & 11.91 & $1.19 \pm 0.04^{\mathrm{d}}$ & 19.55 & $0.30 \pm 0.01^{\mathrm{bcd}}$ & 22.89 \\
\hline Bapa & 30 & $11.32 \pm 0.16^{\mathrm{abc}}$ & 14.11 & $7.13 \pm 0.10^{\mathrm{b}}$ & 7.90 & $1.54 \pm 0.03^{\mathrm{abc}}$ & 12.25 & $1.37 \pm 0.03^{\mathrm{bc}}$ & 15.03 & $0.30 \pm 0.01^{\mathrm{abcd}}$ & 21.43 \\
\hline Sekakouo & 30 & $11.54 \pm 0.29^{\mathrm{ab}}$ & 10.24 & $7.00 \pm 0.11^{\mathrm{bcd}}$ & 8.69 & $1.46 \pm 0.05^{\mathrm{bc}}$ & 21.49 & $1.38 \pm 0.03^{\mathrm{bc}}$ & 14.41 & $0.27 \pm 0.01^{\text {def }}$ & 20.60 \\
\hline Bangoulap & 30 & $11.59 \pm 0.15^{\mathrm{ab}}$ & 7.40 & $6.70 \pm 0.08^{\mathrm{cd}}$ & 6.71 & $1.43 \pm 0.02^{\mathrm{c}}$ & 10.51 & $1.37 \pm 0.03^{\mathrm{bc}}$ & 15.67 & $0.28 \pm 0.00^{\text {bcdef }}$ & 20.74 \\
\hline Foumban & 30 & $11.06 \pm 0.14^{\mathrm{bcd}}$ & 7.22 & $6.28 \pm 0.10^{\mathrm{f}}$ & 9.37 & $1.27 \pm 0.05^{\mathrm{d}}$ & 23.42 & $1.22 \pm 0.03^{\mathrm{d}}$ & 17.62 & $0.27 \pm 0.00^{\operatorname{def}}$ & 13.59 \\
\hline Mbouo & 30 & $10.99 \pm 0.14^{\mathrm{bcd}}$ & 7.44 & $6.37 \pm 0.10^{\mathrm{cf}}$ & 8.78 & $1.42 \pm 0.02^{\mathrm{c}}$ & 11.51 & $1.25 \pm 0.03^{\mathrm{cd}}$ & 16.91 & $0.25 \pm 0.01^{\mathrm{f}}$ & 24.73 \\
\hline Mean & & $10.98 \pm 0.06$ & 12.49 & $6.71 \pm 0.03$ & 11.17 & $1.48 \pm 0.01$ & 16.82 & $1.32 \pm 0.01$ & 20.51 & $0.29 \pm 0.00$ & 22.58 \\
\hline
\end{tabular}

$a, b, c, d, e, f:$ the affected averages of the same letter on the same column do not differ significantly at the $5 \%$ threshold. $\overline{\boldsymbol{X}}_{ \pm}$S.E: Average and standard error. CV: Coefficient of variation. N: Sample size. (Lb): Length of honey bees; (Lab): Length of abdomen; (Wyb): Width of yellow bands on the $2^{\text {nd }}$ abdominal tergite; (Wab): Width of $4^{\text {th }}$ abdominal tergite; (Lh): Length of hairiness of the hairy zone on the 5 th abdominal tergite

Table 1 (cnt'd): Number, mean values and coefficients of variation of honey bee morpho-metric characteristics according to locations in the Western Highlands of Cameroon

\begin{tabular}{|c|c|c|c|c|c|c|c|c|c|c|}
\hline \multirow[b]{2}{*}{ Localities } & \multicolumn{2}{|r|}{$\mathrm{La}(\mathrm{mm})$} & \multicolumn{2}{|r|}{ Lp (mm) } & \multicolumn{2}{|c|}{$\mathrm{A}(\mathrm{mm})$} & \multicolumn{2}{|c|}{ B (mm) } & \multicolumn{2}{|c|}{ Ds (mm) } \\
\hline & $\mathrm{N}$ & $\overline{\boldsymbol{X}}_{ \pm \text {S.E }}$ & $\mathrm{CV}(\%)$ & $\overline{\boldsymbol{X}}_{ \pm \text {S.E }}$ & $\mathrm{CV}(\%)$ & $\overline{\boldsymbol{X}}_{ \pm \text {S.E }}$ & $\mathrm{CV}(\%)$ & $\overline{\boldsymbol{X}}_{ \pm \text {S.E }}$ & $\mathrm{CV}(\%)$ & $\overline{\boldsymbol{X}}_{ \pm \text {S.E }}$ \\
\hline $\begin{array}{l}\text { Santchou } \\
\end{array}$ & 30 & $4.24 \pm 0.03^{\text {abcd }}$ & 2.61 & $2.00 \pm 0.26^{\mathrm{e}}$ & 29.66 & $0.57 \pm 0.02^{\mathrm{bc}}$ & 11.21 & $0.23 \pm 0.00^{\mathrm{a}}$ & 11.35 & $-1.36 \pm 0.03^{\mathrm{bc}}$ \\
\hline Melia & 30 & $4.03 \pm 0.05^{\mathrm{de}}$ & 4.82 & $2.97 \pm 0.23^{\mathrm{d}}$ & 26.49 & $0.60 \pm 0.03^{\mathrm{bc}}$ & 21.41 & $0.25 \pm 0.01^{\mathrm{a}}$ & 13.16 & $-0.08 \pm 0.05^{\mathrm{b}}$ \\
\hline Dschang & 30 & $4.35 \pm 0.08^{\mathrm{abc}}$ & 6.47 & $3.71 \pm 0.18^{\mathrm{c}}$ & 15.03 & $0.61 \pm 0.01^{\mathrm{bc}}$ & 8.29 & $0.25 \pm 0.01^{\mathrm{a}}$ & 18.21 & $0.05 \pm 0.03^{\mathrm{a}}$ \\
\hline Bamepa & 30 & $4.48 \pm 0.05^{\mathrm{a}}$ & 3.57 & $4.70 \pm 0.34^{\mathrm{b}}$ & 17.75 & $0.58 \pm 0.01^{\mathrm{bc}}$ & 5.51 & $0.26 \pm 0.01^{\mathrm{a}}$ & 14.00 & $-0.20 \pm 0.03^{\text {bc }}$ \\
\hline Bamessingue & 30 & $4.38 \pm 0.06^{\mathrm{abc}}$ & 4.83 & $3.65 \pm 0.18^{c}$ & 15.96 & $0.63 \pm 0.01^{\mathrm{ab}}$ & 6.39 & $0.23 \pm 0.01^{\mathrm{a}}$ & 17.21 & $-0.11 \pm 0.02^{\mathrm{b}}$ \\
\hline Santa & 30 & $4.19 \pm 0.12^{\mathrm{bcd}}$ & 9.47 & $3.54 \pm 0.22^{\mathrm{cd}}$ & 17.06 & $0.61 \pm 0.01^{\mathrm{bc}}$ & 9.75 & $0.27 \pm 0.01^{\mathrm{a}}$ & 12.04 & $-0.15 \pm 0.02^{\mathrm{bc}}$ \\
\hline Famla'a & 30 & $4.46 \pm 0.05^{\mathrm{a}}$ & 3.85 & $5.05 \pm 0.27^{\mathrm{ab}}$ & 12.09 & $0.60 \pm 0.01^{\mathrm{bc}}$ & 7.23 & $0.25 \pm 0.01^{\mathrm{a}}$ & 16.94 & $-0.19 \pm 0.03^{\text {bc }}$ \\
\hline Koutaba & 30 & $4.15 \pm 0.07^{\text {cd }}$ & 5.72 & $5.04 \pm 0.19^{\mathrm{ab}}$ & 9.98 & $0.61 \pm 0.01^{\mathrm{bc}}$ & 8.20 & $0.26 \pm 0.01^{\mathrm{a}}$ & 12.04 & $-0.08 \pm 0.01^{\mathrm{b}}$ \\
\hline Banka & 30 & $4.33 \pm 0.06^{\mathrm{abc}}$ & 5.03 & $5.58 \pm 0.13^{\mathrm{a}}$ & 6.65 & $0.61 \pm 0.00^{\mathrm{bc}}$ & 5.09 & $0.28 \pm 0.00^{\mathrm{a}}$ & 13.83 & $-0.24 \pm 0.02^{c}$ \\
\hline Bapa & 30 & $4.41 \pm 0.03^{\mathrm{ab}}$ & 2.26 & $4.76 \pm 0.43^{b}$ & 20.51 & $0.60 \pm 0.02^{\mathrm{bc}}$ & 11.25 & $0.28 \pm 0.01^{\mathrm{a}}$ & 11.55 & $-0.14 \pm 0.03^{\text {bc }}$ \\
\hline Sekakouo & 30 & $4.37 \pm 0.03^{\mathrm{abc}}$ & 2.76 & $5.37 \pm 0.14^{\mathrm{ab}}$ & 6.94 & $0.67 \pm 0.01^{\mathrm{a}}$ & 8.68 & $0.26 \pm 0.01^{\mathrm{a}}$ & 13.37 & $-0.10 \pm 0.03^{b}$ \\
\hline Bangoulap & 30 & $4.29 \pm 0.06^{\mathrm{abc}}$ & 5.00 & $5.12 \pm 0.21^{\mathrm{ab}}$ & 11.18 & $0.60 \pm 0.01^{\mathrm{bc}}$ & 8.31 & $0.26 \pm 0.00^{\mathrm{a}}$ & 9.39 & $-0.16 \pm 0.04^{\text {bc }}$ \\
\hline Foumban & 30 & $4.24 \pm 0.07^{\mathrm{abcd}}$ & 5.94 & $5.16 \pm 0.18^{\mathrm{ab}}$ & 10.24 & $0.55 \pm 0.01^{\mathrm{cd}}$ & 6.52 & $0.25 \pm 0.00^{\mathrm{a}}$ & 11.28 & $-0.20 \pm 0.04^{\mathrm{bc}}$ \\
\hline Mbouo & 30 & $3.93 \pm 0.12^{\mathrm{e}}$ & 10.13 & $5.19 \pm 0.13^{\mathrm{ab}}$ & 8.49 & $0.50 \pm 0.01^{\mathrm{d}}$ & 10.93 & $0.24 \pm 0.01^{\mathrm{a}}$ & 18.70 & $-0.09 \pm 0.05^{\mathrm{b}}$ \\
\hline Mean & & $4.27 \pm 0,02$ & 6.40 & $4.39 \pm 0.11$ & 26.19 & $0.60 \pm 0.00$ & 11.53 & $0.25 \pm 0.00$ & 14.29 & $-0.13 \pm 0.01$ \\
\hline
\end{tabular}

a, b, c, d, e, f: the affected averages of the same letter on the same column do not differ significantly at the 5\% threshold. $\overline{\boldsymbol{X}}_{ \pm}$S.E: Average and standard error. CV: Coefficient of variation.

N: Sample size. (La): Length of antennas; (Lp): length of proboscis; (A): length of nervure A; (B): length of nervure B; (Ds): Discoidal shift 
Fotso et al, 2021. Gen. Biodiv. J: 5 (2): 1-11

Table 1 (cnt'd): Number, mean values and coefficients of variation of honey bee morpho-metric characteristics according to locations in the Western Highlands of Cameroon

\begin{tabular}{|c|c|c|c|c|c|c|c|c|c|}
\hline \multirow[b]{2}{*}{ Localities } & \multirow[b]{2}{*}{$\mathrm{N}$} & \multirow{2}{*}{$\begin{array}{c}\text { Lraw (mm) } \\
\overline{\boldsymbol{X}}_{ \pm \text {S.E }}\end{array}$} & \multicolumn{3}{|c|}{ Raw (mm) } & \multirow{2}{*}{$\frac{\text { Lrpw (mm) }}{\overline{\boldsymbol{X}}_{ \pm \text {S.E }}}$} & \multicolumn{3}{|c|}{ Wrpw (mm) } \\
\hline & & & $\mathrm{CV}(\%)$ & $\overline{\boldsymbol{X}}_{ \pm \mathrm{S} . \mathrm{E}}$ & $\mathrm{CV}(\%)$ & & CV (\%) & $\overline{\boldsymbol{X}}_{ \pm \text {S.E }}$ & $\mathrm{CV}(\%)$ \\
\hline Santchou & 30 & $9.17 \pm 0.11^{\mathrm{bc}}$ & 3.82 & $3.03 \pm 0.05^{\mathrm{de}}$ & 6.08 & $6.26 \pm 0.07^{\mathrm{bc}}$ & 3.76 & $1.65 \pm 0.03^{\mathrm{abc}}$ & 6.84 \\
\hline Melia & 30 & $8.50 \pm 0.12^{\mathrm{d}}$ & 5.04 & $2.93 \pm 0.04^{\mathrm{ef}}$ & 5.34 & $5.71 \pm 0.18^{\mathrm{d}}$ & 10.88 & $1.57 \pm 0.07^{\mathrm{cd}}$ & 16.22 \\
\hline Dschang & 30 & $9.48 \pm 0.07^{\mathrm{ab}}$ & 2.62 & $3.30 \pm 0.03^{\mathrm{a}}$ & 3.08 & $6.54 \pm 0.08^{\mathrm{abc}}$ & 4.11 & $1.79 \pm 0.04^{\mathrm{ab}}$ & 8.26 \\
\hline Bamepa & 30 & $9.38 \pm 0.09^{\mathrm{ab}}$ & 3.19 & $3.20 \pm 0.04^{\mathrm{abc}}$ & 4.13 & $6.37 \pm 0.10^{\mathrm{abc}}$ & 5.00 & $1.67 \pm 0.03^{\mathrm{abc}}$ & 7.39 \\
\hline Bamessingue & 30 & $9.49 \pm 0.09^{\mathrm{ab}}$ & 3.21 & $3.29 \pm 0.05^{\mathrm{a}}$ & 4.87 & $6.52 \pm 0.10^{\mathrm{abc}}$ & 5.24 & $1.73 \pm 0.06 \mathrm{abc}$ & 12.52 \\
\hline Santa & 30 & $9.22 \pm 0.08^{\mathrm{bc}}$ & 2.80 & $3.20 \pm 0.02^{\mathrm{abc}}$ & 1.99 & $6.37 \pm 0.08^{\mathrm{abc}}$ & 4.03 & $1.67 \pm 0.04^{\mathrm{abc}}$ & 9.21 \\
\hline Famla'a & 30 & $9.45 \pm 0.09^{\mathrm{ab}}$ & 3.26 & $3.24 \pm 0.03^{\mathrm{abc}}$ & 3.56 & $6.58 \pm 0.07^{\mathrm{ab}}$ & 3.30 & $1.80 \pm 0.02^{\mathrm{a}}$ & 4.80 \\
\hline Koutaba & 30 & $9.19 \pm 0.09^{\mathrm{bc}}$ & 3.15 & $3.12 \pm 0.04^{\mathrm{bcd}}$ & 4.19 & $6.40 \pm 0.05^{\mathrm{abc}}$ & 2.84 & $1.74 \pm 0.02^{\mathrm{ab}}$ & 4.64 \\
\hline Banka & 30 & $9.51 \pm 0.11^{\mathrm{ab}}$ & 3.75 & $3.16 \pm 0.01^{\mathrm{abcd}}$ & 1.46 & $6.44 \pm 0.05^{\mathrm{abc}}$ & 2.89 & $1.71 \pm 0.03^{\mathrm{abc}}$ & 7.30 \\
\hline Bapa & 30 & $9.68 \pm 0.14^{\mathrm{a}}$ & 4.86 & $3.17 \pm 0.04^{\mathrm{abcd}}$ & 4.56 & $6.62 \pm 0.06^{\mathrm{a}}$ & 2.97 & $1.66 \pm 0.05^{\mathrm{abc}}$ & 10.00 \\
\hline Sekakouo & 30 & $9.73 \pm 0.07^{\mathrm{a}}$ & 2.32 & $3.27 \pm 0.04^{\mathrm{bc}}$ & 4.43 & $6.52 \pm 0.07^{\mathrm{abc}}$ & 3.67 & $1.79 \pm 0.05^{\mathrm{ab}}$ & 10.22 \\
\hline Bangoulap & 30 & $9.52 \pm 0.14^{\mathrm{ab}}$ & 4.76 & $3.23 \pm 0.03^{\mathrm{abc}}$ & 3.86 & $6.57 \pm 0.09^{\mathrm{ab}}$ & 4.44 & $1.73 \pm 0.05^{\mathrm{abc}}$ & 9.38 \\
\hline Foumban & 30 & $9.18 \pm 0.13^{\mathrm{bc}}$ & 4.66 & $3.09 \pm 0.05^{\mathrm{cd}}$ & 5.22 & $6.37 \pm 0.09^{\mathrm{abc}}$ & 4.87 & $1.63 \pm 0.04^{\mathrm{bcd}}$ & 7.87 \\
\hline Mbouo & 30 & $8.94 \pm 0.16^{\mathrm{c}}$ & 5.76 & $2.81 \pm 0.09^{f}$ & 10.16 & $6.20 \pm 0.15^{\mathrm{c}}$ & 8.01 & $1.48 \pm 0.06^{\mathrm{d}}$ & 12.82 \\
\hline Mean & & $9.31 \pm 0.03$ & 5.03 & $3.14 \pm 0.01$ & 6.27 & $6.38 \pm 0.03$ & 6.03 & $1.69 \pm 0.01$ & 10.42 \\
\hline
\end{tabular}

a, b, c, d, e, f: the affected averages of the same letter on the same column do not differ significantly at the 5\% threshold. $\overline{\boldsymbol{X}} \pm$ S.E: Average and standard error. CV: Coefficient of variation. N: Sample size. Lraw: Length of right anterior wings. Raw: Width of right anterior wings, Lrpw: Length of right posterior wings. Wrpw: Width of right posterior wings

Table 2: Correlations between body measurements

\begin{tabular}{|c|c|c|c|c|c|c|c|c|c|c|c|c|c|c|}
\hline & $\mathrm{Lb}$ & $\mathrm{Lab}$ & Wyb & Wab & $\mathrm{Lh}$ & Laba & $\mathrm{Lp}$ & $\mathrm{A}$ & $\mathrm{B}$ & Lraw & Raw & Ds & Lrpw & Wrpw \\
\hline $\mathrm{Lb}$ & 1 & & & & & & & & & & & & & \\
\hline $\mathrm{Lab}$ & $0.43^{* *}$ & 1 & & & & & & & & & & & & \\
\hline Wyb & 0.04 & $0.18^{* *}$ & 1 & & & & & & & & & & & \\
\hline Wab & $0.28^{* * *}$ & $0.66^{* * *}$ & 0.08 & 1 & & & & & & & & & & \\
\hline Lh & -0.01 & $0.17^{* *}$ & $0.14^{* *}$ & 0.01 & 1 & & & & & & & & & \\
\hline Laba & $0.19^{*}$ & $0.29^{* *}$ & $0.24^{* *}$ & $0.24^{* *}$ & $0.22^{* *}$ & 1 & & & & & & & & \\
\hline Lp & $0.41^{* *}$ & 0.03 & 0.09 & -0.04 & -0.16 & 0.04 & 1 & & & & & & & \\
\hline A & 0.01 & 0.04 & $0.17^{*}$ & 0.14 & 0.13 & $0.20^{*}$ & -0.04 & 1 & & & & & & \\
\hline B & 0.11 & 0.11 & -0.09 & 0.06 & -0.01 & 0.15 & 0.18 & 0.13 & 1 & & & & & \\
\hline Lraw & $0.33^{* *}$ & $0.37^{* *}$ & $0.25^{* *}$ & $0.22^{* *}$ & $0.20^{* *}$ & $0.44^{* *}$ & $0.30^{* *}$ & $0.30^{* *}$ & $0.06^{* *}$ & 1 & & & & \\
\hline Raw & 0.12 & $0.34^{* *}$ & $0.23^{* *}$ & $0.28^{* *}$ & 0.14 & $0.48^{* *}$ & 0.10 & $0.43^{* *}$ & 0.05 & $0.60^{* *}$ & 1 & & & \\
\hline Ds & -0.12 & 0.11 & 0.02 & 0.07 & 0.12 & -0.14 & -0.19 & 0.01 & 0.01 & 0.02 & -0.02 & 1 & & \\
\hline Lrpw & $0.30^{* *}$ & $0.41^{* *}$ & $0.31^{* *}$ & $0.24^{* *}$ & 0.13 & $0.31^{* *}$ & $0.25^{*}$ & 0.15 & 0.08 & $0.72^{* *}$ & $0.51^{* *}$ & 0.03 & 1 & \\
\hline Wrpw & 0.11 & $0.32^{* *}$ & 0.13 & 0.17 & $0.21^{*}$ & $0.30^{* *}$ & -0.03 & $0.18^{*}$ & -0.03 & $0.28^{* *}$ & $0.42^{* * *}$ & 0.01 & $0.26^{* *}$ & 1 \\
\hline
\end{tabular}

(**): $\mathrm{p}<0.01 ;(*): \mathrm{p}<0.05$; (Lb): Length of honey bee; (Lab): Length of Abdomen; (Wyb): Width of yellow bands on the 2 nd abdominal tergite; (Wab): Width of 4 il abdominal tergite; (Lh): Length of hairiness of the hairy zone on the $5^{\text {th }}$ abdominal tergite; (La): Length of antennas; (Lp): length of proboscis; (A): length of nervure A; (B): length of nervure B; (Lraw): Length of right anterior wing; (Raw): Width of right anterior wing; (Ds): Discoidal shift; (Lrpw): Length of right posterior wings; (Wrpw): Width of right posterior wing 
The mean length of honeybees in the localities of Banka (11.98 \pm 0.16$)$ and Famla'a (11.87 \pm 0.27$)$ are statistically identical but greater $(p<0.05)$ than that of honeybees from Melia, Dschang, Bamepa, Santa and Santchou. The coefficient of variation of this measurement varies from 7.22 to $14.5 \%$ and the honeybees are mostly heterogeneous in the locality of Melia $(\mathrm{CV}=14.50 \%)$ for those traits.

The average length of nervure A of honeybees is $0.60 \pm 0.00 \mathrm{~mm}$, with a coefficient of variation of 11.53. The average length of nervure $A$ in Mbouo and Feubant localities while remaining statistically identical $(p \geq 0.05)$ is significantly lower $(p<0.05)$ than honeybees in other localities. The highest coefficient of variation $(\mathrm{CV}=21.41 \%)$ is observed in Melia locality.

The average length of nervure $B$ of honeybees is $0.25 \pm 0.00 \mathrm{~mm}$, with a coefficient of variation of 14.29. Taking into account each locality, the averages are statistically comparable $(p \geq 0.05)$. The highest coefficient of variation $(\mathrm{CV}=18.70 \%)$ is observed in Mbouo local honeybees.

Correlations coefficients between body measurements of honeybees in the Western Highlands of Cameroon

Table 2 presents the correlation coefficients between body measurements of honeybees in the Western Highlands of Cameroon.

The significantly higher correlation coefficient (0.72) is obtained between the length of the right hindwing and the length of the right forewing, and the lowest is obtained between the discoidal shift and the length of the proboscis (-0.19).

\section{Cubital index of honeybees in the Western Highlands Cameroon}

Table 3: Sample size, means, and coefficients of variation of the cubital index (CI) of honeybees by location in the Western Highlands of Cameroon

Table 3: Sample size, means, and coefficients of variation of the cubital index (CI)

\begin{tabular}{|c|c|c|c|}
\hline \multirow[b]{2}{*}{ Localities } & \multirow[b]{2}{*}{$\mathrm{N}$} & \multicolumn{2}{|c|}{$\mathrm{CI}$} \\
\hline & & $\overline{\boldsymbol{X}} \pm$ S.E & $\mathrm{CV}(\%)$ \\
\hline Santchou & 30 & $2.49 \pm 0.14^{\mathrm{abc}}$ & 17.16 \\
\hline Melia & 30 & $2.34 \pm 0.15^{\mathrm{abc}}$ & 21.47 \\
\hline Dschang & 30 & $2.49 \pm 0.12^{\mathrm{abc}}$ & 15.35 \\
\hline Bamepa & 30 & $2.20 \pm 0.11^{b c}$ & 15.58 \\
\hline Bamessingue & 30 & $2.75 \pm 0.15^{\mathrm{a}}$ & 17.43 \\
\hline Santa & 30 & $2.29 \pm 0.13^{\mathrm{bc}}$ & 17.68 \\
\hline Famla'a & 30 & $2.42 \pm 0.19^{\mathrm{abc}}$ & 24.59 \\
\hline Koutaba & 30 & $2.34 \pm 0.13^{\mathrm{abc}}$ & 18.16 \\
\hline Banka & 30 & $2.22 \pm 0.13^{\mathrm{bc}}$ & 17.91 \\
\hline Bapa & 30 & $2.18 \pm 0.11^{\mathrm{bc}}$ & 15.65 \\
\hline Sekakouo & 30 & $2.62 \pm 0.13^{\mathrm{ab}}$ & 16.20 \\
\hline Bangoulap & 30 & $2.36 \pm 0.10^{\mathrm{abc}}$ & 13.73 \\
\hline Foumban & 30 & $2.21 \pm 0.07^{\mathrm{bc}}$ & 9.86 \\
\hline Mbouo & 30 & $2.10 \pm 0.15^{\mathrm{c}}$ & 23.11 \\
\hline Mean & & $2.36 \pm 0.04$ & 18.55 \\
\hline
\end{tabular}

a, b, c, the affected averages of the same letter on the same column do not differ significantly at the 5\% threshold. $\overline{\boldsymbol{X}}_{ \pm}$S.E: Average and standard error. CV: Coefficient of variation. N: sample size. CI: Cubital Index

The average honeybees' cubital index (Table 3) is $2.36 \pm 0.04$ with a coefficient of variation of $18.55 \%$. The significantly lower average cubital index is obtained in honeybees from Mbouo locality $(2.10 \pm 0.15)$. Between localities, the coefficient of variation varies from 9.86 to $24.59 \%$. The most heterogeneous honeybees are found in the locality of Famla'a $(\mathrm{CV}=24.59 \%)$.

\section{Analysis of the genetic variability of honey bee populations in the Western Highlands of Cameroon}

The principal component analysis (PCA) was performed on the basis of the 14 quantitative variables measured in honeybees which are: honey bee length, length of abdomen, width of yellow bands on the $2^{\text {nd }}$ abdominal tergite, width of $4^{\text {th }}$ abdominal tergite, length of hairiness of hairy zone on $5^{\text {th }}$ abdominal 
tergite, length of the antenna, length of proboscis, length of nervure A, length of nervure B, length of right anterior wing, width of right anterior wing, discoidal displacement, length of right posterior wing and width of right posterior wing.

Table 4 presents the contribution of the 14 main components to the observed genetic variability among honey bee populations in the Western Highlands of Cameroon.

The first 12 components explain $97.13 \%$ of the genetic variability observed in the honey bee population studied. However, it should be noted that the first, second and third are the major components and account for $51.01 \%$ of the total variability observed in the honey bee population studied.

\section{Structure of honey bee populations in the Western Highlands of Cameroon}

The discriminant factor analysis enable us to determine the genetic types of honeybees on the Western Highlands of Cameroon.

Figure 1 illustrates the structure of the honey bee population observed in the Western Highlands of Cameroon.

Table 4: Contribution of the 14 main components to the genetic variability observed in honey bee populations in the Western Highlands of Cameroon

\begin{tabular}{lccc}
\hline Principal component & Eigenvalue & Variability $(\%)$ & Cumulative variability \\
\hline PC1 & 4.15 & 29.66 & 29.66 \\
PC2 & 1.67 & 11.94 & 41.60 \\
PC3 & 1.32 & 9.42 & 51.01 \\
PC4 & 1.13 & 8.06 & 59.08 \\
PC5 & 1.04 & 7.43 & 66.50 \\
PC6 & 0.93 & 6.67 & 73.17 \\
PC7 & 0.83 & 5.95 & 79.12 \\
PC8 & 0.69 & 4.95 & 84.07 \\
PC9 & 0.62 & 4.44 & 88.52 \\
PC10 & 0.48 & 3.39 & 91.91 \\
PC11 & 0.41 & 2.95 & 94.86 \\
PC12 & 0.32 & 2.27 & 97.13 \\
PC13 & 0.20 & 1.44 & 98.58 \\
PC14 & 0.20 & 1.42 & 100.00 \\
\hline
\end{tabular}

PC: Principal component

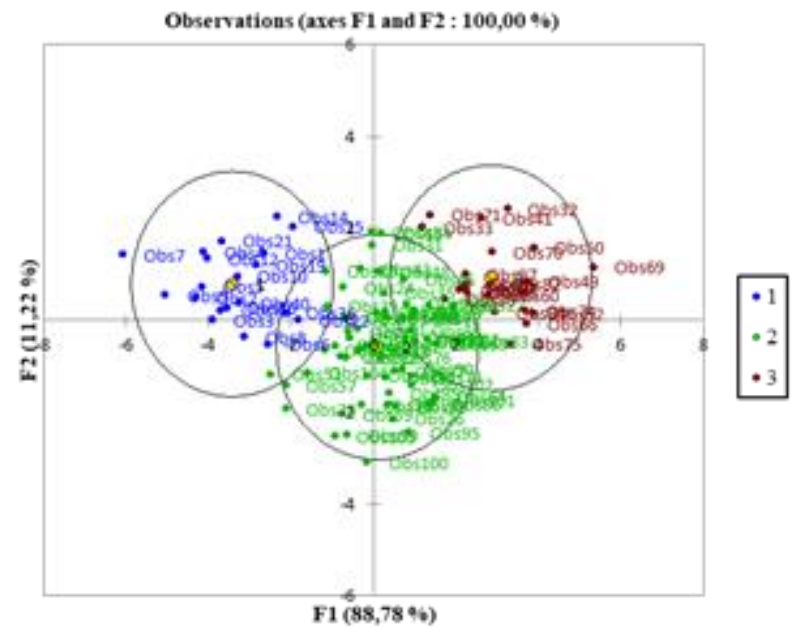

1: Genetic type $1 ; 2$ : Genetic type 2; 3: Genetic type 3

Figure 1: Structure of the honey bee population in the Western Highlands of Cameroon 
Figure 1 shows that honeybee populations in the Western Highlands of Cameroon consist of three genetic types: genetic type 1, genetic type 2, genetic type 3 .

Figure 2 illustrates a cloud of points produced on a graph where the cubital index is plotted as a function of the discoidal shift; each point represents a honey bee of the sample.

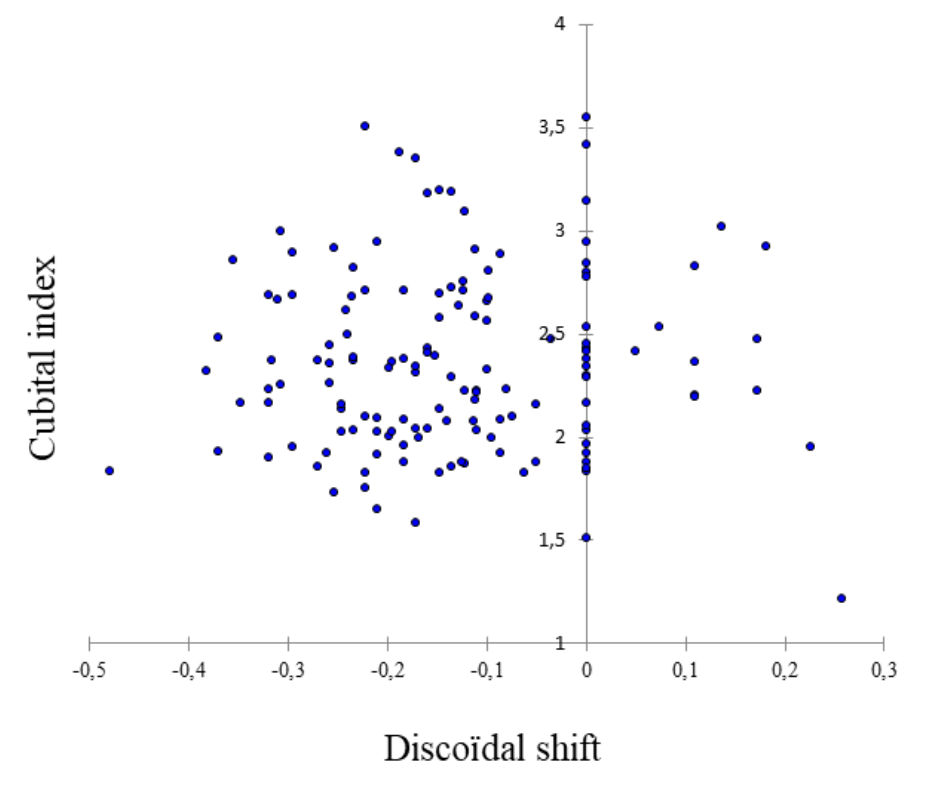

Figure 2: Dots plot between cubital index and discoidal shift of the honey bee population in the Western Highlands of Cameroon

It was observed from Figure 2 that some cubital indices were higher while other discoidal displacements were negative, positive or null, these are mixed colonies. There are three genetic types within the populations of honeybees in the Western Highlands of Cameroon with specific characteristics. Thus, on this basis coupled to figure 1 there is genetic type 1 , with negative discoidal displacement; genetic type 2, with zero discoidal displacement and genetic type 3 with positive discoidal displacement.

Phylogenetic relationship between the genetic types of honeybees of the Western Highlands of Cameroon

Table 5 shows the Euclidean genetic distance between the genetic types of honeybees in the Western Highlands of Cameroon.

Table 5: Euclidean genetic distance between the genetic types of honey bee in the Western Highlands of Cameroon

\begin{tabular}{llll}
\hline & $\mathrm{T} 1$ & $\mathrm{~T} 2$ & $\mathrm{~T} 3$ \\
\hline $\mathrm{T} 1$ & 0 & & \\
$\mathrm{~T} 2$ & 2.72 & 0 & \\
$\mathrm{~T} 3$ & 4.59 & 2.23 & 0 \\
\hline
\end{tabular}

The highest genetic distance (4.59) is obtained between genetic type 3 and genetic type 1, while the smallest genetic distance (2.23) is obtained between genetic type 3 and 2. The intra-genetic variability of honeybees in the Western Highlands of Cameroon is higher (52.92\%) compared to the inter-genetic variability $(47.08 \%)$.

Figure 3 illustrates the phylogenetic tree of honey bee populations in the Western Highlands of Cameroon. 


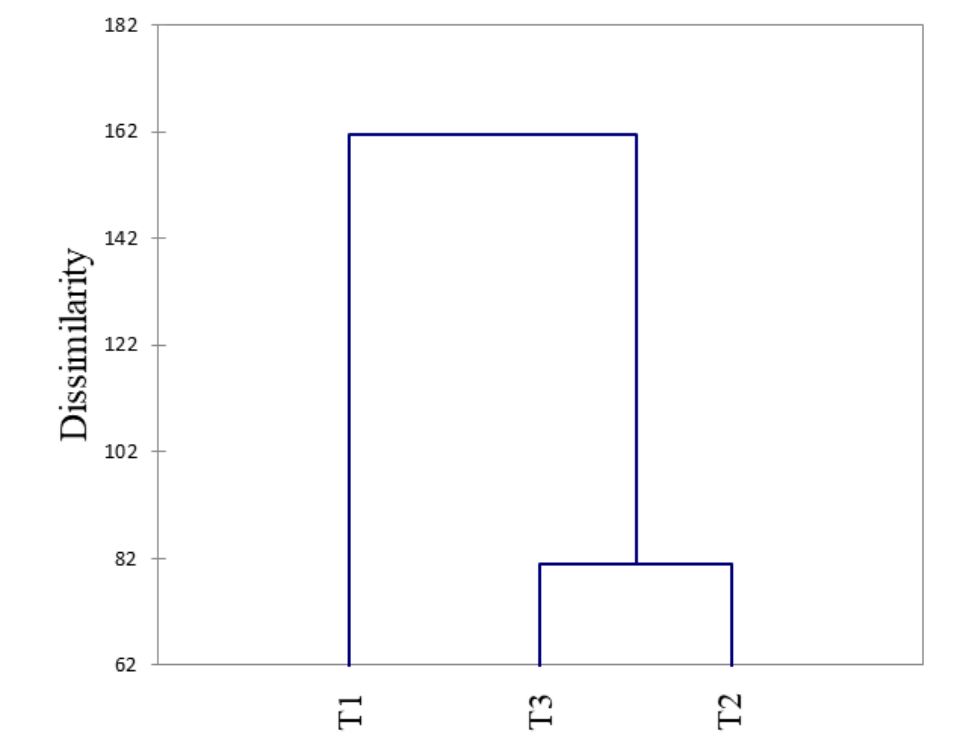

T1: Genetic type 1; T2: Genetic type 2; T3: Genetic type 3

Figure 3: Phylogenetic dendrogram of honeybees' populations in the Western Highlands of Cameroon

Figure 3 establishes the existing genetic relationship between the three identified genetic types. The genetic types 3 and 2 have a very large number of alleles in common and could therefore have a common origin or belong to the same subgroup. Genetic types 1 and 2 are very distant and seem to have very few alleles in common.

\section{Discussion}

With regard to the length of honeybees, our results are $9.02 \%$ lower than those reported by Paraïso et al. (2011) in North-East Benin. This difference could be explained by the genetic composition between populations and the environmental factors to which the bee populations have been subjected.

With respect to the length of nervures $A$ and $B$, our results are comparable $(p \geq 0.05)$ to those obtained by Gadbin et al. (1979). On the other hand, but superior to those reported by Achou (2007) in eastern Algeria and Bendjedid (2010) in southern Algeria.

The discoidal displacement obtained was negative, null or positive. From the work of Toullec (2008), the black honeybees had a negative transgression; it was found positive among Italian and Carniolionnes bees. From the work of Paraïso et al. (2011) in North-East Benin, the honeybees had a negative or null discoidal displacement. This is further proof of the heterogeneity of our bee population, suggesting that there would be subspecies other than Apis mellifera adansonii in the Western Highlands of Cameroon. This information is essential for genetic improvement in honeybees in the study zone.

As for the length and width of the right hindwing, our results are in agreement with those found in eastern Algeria by Achou (2007) and different from those obtained in southern Algeria by Bendjedid (2010) and in north east of Benin by Paraïso et al. (2011). Indeed, the size of the honey bee, the length and width of the large forewing, the length and width of the right hind wing of honeybees and other morphometric characters may vary depending on the environment and other abiotic factors as reported by Fresnaye (1981) and Paraïso et al. (2011). This observation corroborates the results of Bendjedid (2010) in southern Algeria and Hadis et al. (2016) in Iran.

Positive correlations coefficients were obtained between the lengths of the anterior and posterior right wings. Bendjedid (2010) obtained similar results in southern Algeria.

The cubital index of honeybees in the Western Highlands of Cameroon was $2.36 \pm 0.04 \mathrm{~mm}$ and varies significantly $(p<0.05)$ according to localities. This result is consistent with that found by Gadbin et al. (1979) in southern Chad, Tchoumboué et al. (2001) in the Highlands of Western Cameroon, 
Achou (2007) in eastern Algeria, Bendjedid (2010) in southern Algeria, Hadis et al. (2016) in Iran, but differs from that reported by Fresnaye (1965), Cornuet et al. (1982, 1988), Kekeçoglu et al. (2007), Hadisœsil et al., (2008), Toullec (2008) and Abou-Shaara (2013). The discrepancies could be due to subspecies as measurements of the cubital index and the discoidal shift are the most accurate indicators, which are specific to each subspecies or ecotype of honeybees as reported by Fresnaye (1965) and Ruttner (1987). A cubital index greater than 2.1 for more than $10 \%$ of the sample or discoidal displacement greater than zero is a sign of miscegenation (Toullec, 2008). This study confirms the diversity of honeybees in the Western Highlands of Cameroon and the existence of most probable hybrid colonies. Further studies in molecular genetics may also confirm the presence of honey bee subspecies other than Apis mellifera adansonii.

\section{Acknowledgments}

The authors express their gratitude to Dr. Abel WADE, director of the National Veterinary Laboratory (LANAVET), Yaoundé Annex and all his staff for the reception and hospitality. They also want to thank the beekeepers for their collaboration.

\section{Author's Contributions}

Fotso designed and implemented the project and wrote the manuscript. Costa-Maia, Keambou, Baenyi and Defang contributed each to the paper write-up at different level; Manjeli, Teguia and. Tchoumboué supervised the project. All authors discussed the results and contributed to the final manuscript.

\section{Conflict of Interest}

The authors declare no conflict no interest.

\section{References}

Abou-Shaara HF 2013. A morphometry map and a new method for honey bee morphometric analysis by using the ArcGIS. Arthropods, 2(4): 189-199

Achou M 2007. Caractérisation morphométrique, biochimique et moléculaire des populations d'abeilles domestiques de l'Est algérien. Effets physiopathologiques de son parasite majeur Varroa destructor. Thèse de Doctorat; Biologie animale, Université Annaba. 87p

Amakpe F 2010. The Biodiversity of the Honey Bees (Apis mellifera adansonii) in the District of Djidja, Republic of Benin. The International Journal of Environmental, Cultural, Economic and Social Sustainability, 6 (6): 90-104.

Bendjedid H 2010. Etude de la Diversité Morphométrique des Populations Domestiques d'Abeilles du Sud et Comparaison avec Celles du Nord-est Algérien. Mémoire de magistère, Faculté des Sciences Département de Biologie Université Badji Mokhtar. 109p

Bouga M. Alaux C. Bienkowska M. Büchler R. Carreck NL. Cauia E. Chlebo R. Dahle B. Dall'Olio R. De la Rúa P 2011. A review of methods for discrimination of honey bee populations as applied to European beekeeping. Journal of Apicultural Research 50:1, 51-84, DOI: 10.3896/IBRA.1.50.1.06.

Carpentier FG 2007. Introduction aux analyses multidimensionnelles. 135P

Carreck NL. Andree M. Brent CS. Cox-Foster D. Dade HA. Ellis JD. Fani Hatjina F. Van Englesdorp D 2011. Standard methods for Apis mellifera anatomy and dissection. Journal of Apicultural Research 52(4).

Cornuet JM. Albisetti J. Mallet N. Fresnaye J 1982. Etude biométrique d'une population d'abeilles Landaises. Apidologie, 13(1): 3-13.

Cornuet JM. Daoudi A. Mohssine EH. Fresnaye J 1988. Etude biométrique de populations d'abeilles Marocaines. Apidolgie, 19(4): 355-366.

Cornuet JM. Fresnaye L. Tassencourt M 1975. Discrimination et classification de populations d'abeilles à partir de caractères biométriques. Apidologie, 6(2) : 145-187.

FAO 2013. Caractérisation phénotypiques des ressources génétiques animales. 151p

Fotso Kenmogne PR. Meutchieye F. Andriamanalina SI. Youbissi A. Tchoumboué J. Pinta JY. Zango P 2014. Caractéristiques socio-économiques et techniques de l'apiculture dans les 
Départements de Bamboutos, Mifi et Menoua (Région de l'Ouest-Cameroun), Livestock Research for Rural Development 26 (12)

Francoy TM. Wittmann D. Drauschke M. Muller S. Steinhage V. Bezerra-Laure MAF. De Jong D. Gonçalves LS 2008. Identification of Africanized honey bees through wing morphometrics: two fast and efficient procedures. Apidologie 39 : 488-494.

Fresnaye J 1965. Etude biométrique de quelques caractères morphologiques de l'abeille noire Française Apis mellfica mellifica. Ann. Abeille, 8(4): p271-283.

Fresnaye J 1981. Biométrie de l'abeille, 2e éd. Echauffour (Orne), Office pour l'Information et la Documentation en Apiculture, $56 \mathrm{p}$

Gadbin C. Cornuet JM. Fresnaye J 1979. Approche biométrique de la variété locale d'Apis mellifera dans le sud Tchadien. Apidologie, 10 (2):138-152.

Hadis G. Javad NR. Mehdi M. 2016. Comparison of standard and geometric morphometric methods for discrimination of honey bees populations (Apis mellifera L.) in Iran. Journal of Entomology and Zoology Studies 4(1): 47-53.

Hadisøesilo S. Raffiudin R. Susanti W. Atmowidi T. Hepburn C. Radloff SE. Fuchs S. Randall HR 2008. Morphometric analysis and biogeography of Apis koschevnikovi Enderlein (1906). Apidologie, 39: 495-503

Kekeçoğlu M. Bouga M. Soysal MI. Harizanis P 2007. Morphometrics as a Tool for the Study of Genetic Variability of Honey Bees. Journal of Tekirdag Agricultural Faculty.

Meixner MD. Pinto MA. Bouga M. Kryger P. Ivanova E. Fuchs S 2013. Standard methods for characterising subspecies and ecotypes of Apis mellifera. Journal of Apicultural Research 52(4).

Oyerinde AA. Dike MC. Banwo OO. Bamaiyi LJ. Adamu RS 2012. Morphometric and Landmark Based Variations of Apis mellifera L. Wings in the Savannah Agro - Ecological Zone of Nigeria. Global Journal of Science Frontier Research Agriculture and Veterinary Sciences 12 (7).

Paraïso A. Viniwanou N. Akossou AYJ. Mensah GA. Abiola W 2011. Caractérisation morphométrique de l'abeille Apis mellifera adansonii au Nord-Est du Bénin. Int. J. Biol. Chem. Sci. 5(1): 331-344.

Roux M 2006. Algorithmes de classification. Université Paul Cézanne Marseille, 108p

Ruttner F 1987. Biogeography and Taxonomy of Honeybees. New York: Springer-verlag; 284 p.

Tchoumboué J. Tchouamo IR. Pinta JY. Njia MN 2001 Caractéristiques socio-économiques et techniques de l'apiculture dans les hauts plateaux de l'Ouest du Cameroun. Tropicultura, 19(3):141-146.

Tofilski A. 2008. Using geometric morphometrics and standard morphometry to discriminate three honeybee subspecies. Apidologie $39: 558-563$.

Toullec ANK 2008. Abeille noire, Apis mellifera mellifera. Historique et sauvegarde. Thèse de Doctorat Vétérinaire. Faculté de Médecine de Créteil. 168 p 